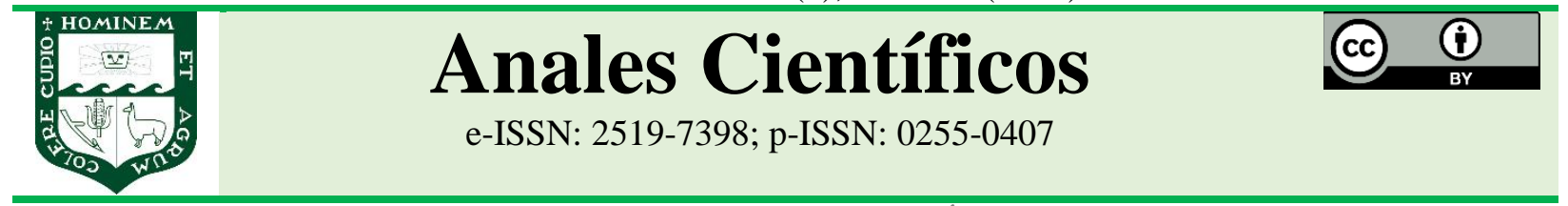

ARTÍCULO ORIGINAL - RESEARCH ARTICLE

http://dx.doi.org/10.21704/ac.v82i2.1765

\title{
CICLO Y PARÁMETROS BIOLÓGICOS DE Clitostethus arcuatus (Coleoptera: Coccinellidae), DEPREDADOR DE MOSCA BLANCA, Siphoninus phillyreae, EN CONDICIONES DE LABORATORIO
}

\section{Cycle and biological parameters of Clitostethus arcuatus (Coleoptera: Coccinellidae), whitefly predator, Siphoninus phillyreae, under laboratory conditions}

\author{
Julissa M. Churata Salcedo'1*(D); Juan G. Mamani Mamani² \\ ${ }^{1}$ Instituto Científico Michael Owen Dillon, Arequipa - Perú. \\ ${ }^{2}$ Laboratorio de Entomología de la Escuela Profesional y Académica de Biología, Facultad de Ciencias Biológicas, \\ Universidad Nacional de San Agustín de Arequipa, Arequipa - Perú. \\ *E-mail: julissa.cs@gmail.com
}

Recibido: 01/06/2020; Aceptado: 02/10/2021; Publicado: 31/12/2021

\begin{abstract}
The objective of this study is to determine biological cycle and parameters of Clitostethus arcuatus (Rossi), predator of Siphoninus phillyreae (Haliday). To obtain prey, olive seedlings were infested with S. phillyreae collected from olive groves in Tacna. In laboratory, the different stages of development of $C$. arcuatus recovered from the same olive groves, were confined in a breeding cage to obtain adults of the same age, which were matched in breeding units, and placed in a stove with circulating air at $24,2 \pm 0,8^{\circ} \mathrm{C}$ and $36,0 \pm 5,9 \% \mathrm{RH}$. They were observed daily until their death, and from their positions the development cycle was determined, which is 24 days, of which 3,80 ; 15,78 and 4,42 days were for the egg, larva and pupa stages, respectively. The biological cycle of $C$. arcuatus was 77,02 days. Oviposition capacity 136,35 eggs/female; egg viability of 71,43\%; larval mortality 36,25\%; predation capacity of LI 3,8 eggs/day, LII 5 eggs/day; LIII 8,2 and 3,6 eggs and nymphs/day respectively, LIV 11,4 and 3,4 eggs and nymphs/day respectively, adults 9,8; 4,6 and 1,4 eggs, nymphs and adults/day respectively; sex ratio 0,59 and longevity 53,02 days.
\end{abstract}

Keywords. Biological control | predator | olive | prey | morphology

\section{RESUMEN}

El objetivo del presente estudio fue determinar el ciclo, parámetros biológicos y descripción morfológica de Clitostethus arcuatus (Rossi), depredador de Siphoninus phillyreae (Haliday). Para la obtención de presas, se infestaron plantones de olivo con S. phillyreae colectado de olivares en Tacna. En el laboratorio, los diferentes estados de desarrollo de $C$. arcuatus fueron recuperados de los mismos olivares, se confinaron en una jaula de crianza para obtener adultos de la misma edad, los cuales fueron emparejados en unidades de crianza (UC), y colocados en una estufa con aire circulante a $24,2 \pm 0,8^{\circ} \mathrm{C}$ y $36,0 \pm 5,9 \% \mathrm{HR}$. Diariamente fueron observados hasta su muerte, y a partir de sus posturas se determinó el ciclo de desarrollo que fue de 24 días, de los cuales 3,80; 15,78 y 4,42 días fueron para los estados de huevo, larva y pupa respectivamente. El ciclo biológico de $C$. arcuatus fue 
de 77,02 días. La capacidad de oviposición 136,35 huevos/hembra; viabilidad de huevos de 71,43\%; mortalidad de larvas 36,25\%; capacidad de depredación de LI 3,8 huevos/día, LII 5 huevos/día; LIII 8,2 y 3,6 huevos y ninfas/día respectivamente, LIV 11,4 y 3,4 huevos y ninfas/día respectivamente, adultos 9,8; 4,6 y 1,4 huevos, ninfas y adultos/día respectivamente; proporción sexual 0,59 y la longevidad de 53,02 días.

Palabras clave: Control biológico | depredador | olivo | presa | morfología

\section{Forma de citar el artículo (Formato APA):}

Churata, J., \& Mamani, J. (2021). Ciclo y parámetros biológicos de Clitostethus arcuatus (Coleoptera: Coccinellidae), depredador de mosca blanca, Siphoninus phillyreae, en condiciones de laboratorio. Anales Científicos. 81(2), 206-218 http://dx.doi.org/10.21704/ac.v82i2.1765

Autor de correspondencia (*) Julissa M. Churata Salcedo. Email: julissa.cs@gmail.com

(C) Los autores. Publicado por la Universidad Nacional Agraria La Molina.

This is an open access article under the CC BY

\section{INTRODUCCIÓN}

Las "moscas blancas" en Perú han alcanzado importancia debido a los problemas que ocasionan en la agricultura (Soria, 2011). Pertenecen al orden Hemiptera: Sternorrhyncha; son pequeñas, poseen alas cubiertas con finas partículas cerosas y blanquecinas. Las especies importantes en Perú son Aleurothrixus floccosus (Maskell) "mosca lanuda de los cítricos" que ataca a frutales cítricos; otras polífagas son Aleurotrachelus trachoides (Back), Trialeurodes vaporariorum (Westwood), Bemisia tabaci (Gennadius); y en Arequipa Siphoninus phillyreae (Haliday) que ataca al árbol ornamental fresno (Osis, 1999).

La "mosca blanca del fresno", Siphoninus phillyreae (Haliday, 1835) (Hemiptera: Aleyrodidae) es una especie del viejo mundo; en América fue detectada por primera vez en California, EE.UU., en 1988, atacando árboles ornamentales y frutales ocasionando pérdidas sustanciales en los cultivos (Bellows et al., 1990). En Chile, el primer reporte fue en 1994 en la Región Metropolitana asociado a Fraxinus excelsior "fresno" (Tapia et al., 2009), dañando a frutales como manzano, pero y cítricos; y otros cultivos de alta producción como olivo. Además, es una seria amenaza para la agricultura en almacigueras de árboles de sombra y cultivos de fruta como ciruelo, almendra, etc. (Bellows et al., 1990). Pedemonte et al. (2009) menciona que $S$. phillyreae es una plaga importante en olivares de las provincias de La Rioja y Catamarca en Argentina porque provoca un debilitamiento general de la planta, caída prematura de hojas y promueve el desarrollo de fumaginas. Valencia (2011) sostiene que S. phillyreae fue reportada para Perú por Arnal y Rondón en 1994 colonizando "granado" (Punica granatum L.) y "fresno" (Fraxinus americana L.); además, Osis (1999) determinó y estudió su ciclo biológico en la zona urbana de Arequipa en $F$. americana. Según SENASA (2010) en marzo del 2009 se detectó a S. phillyreae infestando olivo en Tacna, desde esta región se diseminó y colonizó diferentes cultivos; en olivo alcanzó un índice de infestación de hasta $64,8 \%$, lo que originó pérdidas económicas considerables y disminución de la calidad del fruto. Ante esta situación, se tomaron medidas para su control como lavados a presión y podas, lográndose disminuir hasta un $24,8 \%$ el grado de infestación. Durante estas evaluaciones se detectó a Clitostethus arcuatus (Rossi, 1794) (Coleoptera: Coccinellidae) "chinita".

C. arcuatus es considerado como uno de los más importantes controladores naturales de S. phillyreae, ya que es un depredador que prefiere especies de Aleyrodidae (Tavadjoh et al., 2010), fue utilizado para controlar mosca blanca en EE.UU. y Argentina (Bellows et al., 1990; Pedemonte et al., 2009; Gasparini et al., 2007). En 1995, el INIA La Cruz - Chile importó de California, EE.UU., la avispita Encarsia inaron y el coccinélido $C$. arcuatus para controlar a $S$. phillyreae, que afectaba severamente árboles $\mathrm{y}$ arbustos ornamentales, debido a la gran cantidad de mielecilla excretada por el insecto (Rodríguez y Rodríguez, 2005). Más tarde, en 2008, se introdujo al Valle de Azapa ambas especies de enemigos naturales (Estay et al., 2009) y un año después se reportó la presencia de $C$. arcuatus en las localidades norteñas de Huasco y Arica (González, 2006).

Almonte et al. (2012) indicó que, en mayo del 2011, en Arequipa se confirmó a $S$. phillyreae como una nueva 
plaga localizada en los distritos de Cocachacra y específicamente en el Sector de Vera Cruz Grande y Punta de Bombón en el Sector de Amoquinto, para lo cual se tomaron medidas para su control y prevención como: podas de aclareo, lavados a presión y liberación de $C$. arcuatus. Para moscas blancas se ha demostrado que la estrategia de usar control químico es ineficaz, debido al gran potencial reproductivo que poseen, la dificultad de los productores para detectar las ninfas pequeñas y translúcidas en las primeras etapas de las infestaciones y los impactos negativos sobre los agentes de control biológico (Mota et al., 2008). En Perú, por la importancia del cultivo de olivo en las regiones sureñas del país, es de urgente necesidad realizar trabajos para el control de S. phillyreae. En Tacna, fue observado a C. arcuatus depredando huevos, ninfas y adultos de $S$. phillyreae; por tanto, para una crianza masal de insectos benéficos es necesario conocer su biología, por tal motivo este estudio tuvo como objetivos: determinar el ciclo biológico y los parámetros biológicos (Capacidad de oviposición, viabilidad de huevos, mortalidad de larvas, capacidad de depredación, proporción sexual y longevidad) de C. arcuatus (Rossi), así como también características de su comportamiento.

\section{MATERIALES Y MÉTODOS}

Este estudio se realizó en el Laboratorio de Entomología de la Escuela Profesional y Académica de Biología de la Universidad Nacional de San Agustín de Arequipa ubicado a $16^{\circ} 24^{\prime} 50,99^{\prime \prime}$ LS, $71^{\circ} 32^{\prime} 01,80^{\prime}$ " LO y a 2327 m.s.n.m., durante los meses de abril a julio del año 2013 a temperatura mensual promedio de 24,2 $\pm 0,8{ }^{\circ} \mathrm{C}$ y humedad relativa mensual promedio de 36,0 $\pm 5,9 \%$.

\section{Registros de temperatura y humedad relativa en laboratorio}

Los factores ambientales registrados fueron temperatura y humedad relativa de la estufa eléctrica con aire circulante BS 50 registrados con un termohigrómetro digital BOECO Model SH - 110 colocado dentro de la estufa.

\section{Crianza de Siphoninus phillyreae}

En un invernadero constituido por un armazón de fierro de $100 \mathrm{~cm}$ de ancho x $160 \mathrm{~cm}$ de largo x $160 \mathrm{~cm}$ de altura, recubierto con una malla Raschel 80, se colocaron 10 plantones de olivo de aproximadamente 1 año de edad y de $90 \mathrm{~cm}$ de altura que fueron transplantados a baldes de plástico de 20 litros para el mejor desarrollo de raíces; además de 6 plantones pequeños de olivo de aproximadamente $30 \mathrm{~cm}$ de altura. Para iniciar la infestación se colocaron sobre los plantones, ramas de olivo infestadas con S. phillyreae provenientes del Sector de Magollo, Tacna.

\section{Obtención del depredador Clitostethus arcuatus}

Se visitaron plantaciones de olivo en las diferentes zonas de la periferia de la ciudad de Tacna, donde fueron reportadas infestaciones por S. phillyreae. La zona escogida fue el fundo "Mendoza", ubicado en el Lateral 25-7D, Sector Magollo $\left(18^{\circ} 06^{\prime} 13,36^{\prime}\right.$ ' S 70¹8'48,03'” O, $310 \mathrm{msnm}$ ), en donde se observó la presencia de $C$. arcuatus depredando $S$. phillyreae. Se colectó $C$. arcuatus, colocando ramas de olivo infestadas dentro de una manga de organza blanca de $100 \mathrm{~cm}$ x $50 \mathrm{~cm}$. Enseguida, se sacudió las ramas para que los individuos de C. arcuatus se desprendan de las hojas de olivo y caigan dentro de la manga. Luego, los individuos obtenidos se colocaron en un recipiente de plástico transparente $(12 \mathrm{~cm}$ de altura $\mathrm{x} 12 \mathrm{~cm}$ de diámetro) y se tapó. También se revisó el envés de hojas de olivo infestadas con mosca blanca, cuando se observó algún estado de desarrollo (larva y/o pupa) del depredador, estas hojas fueron colectadas manualmente. Las colectas se realizaron una vez por mes, durante 4 meses y el material colectado era trasladado al laboratorio. Los individuos capturados muertos fueron colocados en alcohol etílico 70\%. Estos individuos fueron identificados utilizando la clave propuesta por González (2012) y también fueron enviados individuos al entomólogo Luis Valencia para la confirmación de la especie, algunos ejemplares fueron entregados al Laboratorio de Entomología de la Universidad Nacional de San Agustín.

\section{Mantenimiento de Clitostethus arcuatus en laboratorio}

En una jaula de crianza instalada en laboratorio se colocaron 6 plantones pequeños de olivo infestados con S. phillyreae, sobre los cuales se colocó las ramas de olivo con individuos de $C$. arcuatus recuperados en campo. Una vez observadas las hojas de olivo con presencia de larvas de $C$. arcuatus, se aislaron y fueron colocadas en placas de Petri de $9,5 \mathrm{~cm}$ de diámetro $\mathrm{x}$ 1,5 de altura adaptadas con organza en la tapa, con el 
suficiente alimento para su desarrollo hasta su empupamiento y la emergencia de los adultos. Se observó el comportamiento de los individuos, sobretodo de la cópula para poder emparejarlos. El alimento fue cambiado, al notarse la falta de turgencia de la hoja y falta de presa, generalmente cada 2-3 días.

\section{Determinación del ciclo biológico de Clitostethus arcuatus}

Los adultos recién emergidos provenientes del proceso anterior, fueron emparejados, obteniendo un total de 20 parejas. Cada pareja fue colocada en un tubo de vidrio de $8 \mathrm{~cm}$ de largo x $2 \mathrm{~cm}$ de diámetro con papel toalla humedecido en su base y una hoja de olivo con suficiente presa; la boca del tubo fue tapada con organza y asegurada con una liguilla. Este conjunto fue considerado como "unidades de crianza" (UC), que fueron colocadas dentro de la estufa con aire circulante BS 50, en la cual se colocó un termohigrómetro BOECO Model SH-110 para registrar la temperatura y humedad relativa. Diariamente se revisó las UC en busca de oviposiciones y cambiar la hoja de olivo si era necesario por escasez de presa. Las hojas de olivo con posturas fueron trasladadas a placas de Petri de $14 \mathrm{~cm}$ de diámetro x 1,5 de altura y en el interior de base de la placa se colocó papel toalla humedecido. Se revisó diariamente la eclosión de los huevos. Después de cada observación se agregó agua usando un gotero al papel toalla puesto en la base de la placa para mantener la humedad, asimismo se aprovechó para realizar la limpieza de la UC.

Para determinar el ciclo de desarrollo se evaluó a 112 huevos obtenidos de las unidades de crianza. En los huevos se determinó el periodo de incubación que consistió en observar desde la puesta de huevos hasta la emergencia de larvas. Seguidamente, se observaron las larvas emergidas hasta su empupamiento y la emergencia de los adultos. Posteriormente, para determinar el ciclo biológico se observó la sobrevivencia de los individuos hasta su muerte. Durante este proceso se obtuvieron las medidas de los estados de desarrollo y su descripción morfológica. Así, de los 112 huevos con que se inició, 20 de ellos fueron medidos considerando su longitud y ancho usando papel milimetrado y un estereoscopio; también se determinó la cantidad de los huevos eclosionados y con este resultado se calculó la viabilidad de huevos. Para la duración del estado de larva y de cada estadío larval, se aislaron 80 larvas recién emergidas en placas de Petri antes descritas y se les suministró suficientes presas, se separó al azar 20 larvas recién emergidas (LI) para medir su longitud y su cápsula cefálica usando un ocular micrométrico; se procedió de la misma manera para los siguientes estadíos larvales.

Se aislaron 20 pupas que llegaron a este para medir su longitud. Se revisaron diariamente para registrar el día de emergencia de los adultos y obtener la duración del estado de pupa, registrando las fechas de emergencia. Se determinaron los parámetros biológicos: capacidad de oviposición, viabilidad de huevos, mortalidad de larvas, capacidad de depredación, proporción sexual y longevidad.

\section{Parámetros biológicos de Clitostethus arcuatus Capacidad de oviposición}

Se formaron 20 parejas, cada pareja fue colocada en una UC, fueron alimentados diariamente con ninfas $\mathrm{y}$ huevos de $S$. phillyreae y se humedeció el papel toalla de la base del tubo. Se observó diariamente para cuantificar los huevos ovipositados.

\section{Viabilidad de huevos}

Calculada a partir 112 huevos obtenidos en las UC. Esta cantidad de posturas fue el total encontrado en la primera semana de observación. Se separó las hojas que contenían un número variable de posturas (5-10 huevos/hoja) y se colocaron en 3 placas de Petri con papel toalla humedecido en la base; cada placa contenía a 33, 39 y 40 huevos/placa, y se revisaron diariamente para registrar el número de larvas emergidas. Se cuantificó los huevos eclosionados para calcular el porcentaje de la viabilidad de huevos.

\section{Mortalidad de larvas}

Del total de huevos aislados, emergieron larvas. Cada larva fue colocada sobre una hoja de olivo con suficiente presa, y cada hoja fue colocada individualmente en recipientes de plástico transparentes de $5,5 \mathrm{~cm}$ de altura $\mathrm{x} 3 \mathrm{~cm}$ de diámetro, con papel toalla humedecido en la base, la boca de los recipientes fue asegurados con organza y una liguilla. A cada larva se le suministró suficiente presa y al observarse escasez de presas sobre las hojas de olivo se trasladó la larva a otra hoja con una mayor cantidad de alimento. Se anotó la cantidad de larvas vivas y muertas de cada estadío larval hasta el inicio del estado de pupa. 


\section{Capacidad de depredación}

Para determinar la capacidad de depredación del estado larval se colocó una larva sobre una hoja de olivo con un número determinado de huevos y ninfas de $S$. phillyreae y fue puesta en una UC. Diariamente se cambió la hoja de olivo, así como se determinó la capacidad de depredación por conteo de las ninfas y huevos que permanecían vivos hasta el día siguiente. Para cada estadío larval y adulto de $C$. arcuatus se realizó 5 repeticiones, a todas se les ofreció huevos, ninfas y adultos de $S$. phillyreae. Esto fue observado con ayuda de un estereoscopio y se anotó los resultados del consumo diario de la presa.

\section{Proporción sexual}

Para la determinación de la proporción sexual se aisló 51 pupas en placas de Petri de 9,5 cm de diámetro x 1,5 de altura adaptadas con organza en la tapa y se esperó hasta la emergencia de los adultos. Para reconocimiento de hembras y machos (sexado), se estudió la morfología externa e interna, la característica más relevante externamente fue el color de cabeza siendo amarilla para machos y negra para hembras, esto fue confirmado con la disecación de genitalia de los individuos.

\section{Longevidad}

La longevidad de los adultos se determinó a partir de 51 individuos adultos que emergieron de las pupas aisladas anteriormente, es decir, el tiempo desde que emergieron hasta su muerte. Estos adultos fueron puestos en placas de Petri de 9,5 cm de diámetro x 1,5 de altura adaptadas con organza en la tapa y papel toalla humedecido en la base, que contenían hojas de olivo con suficiente presa.

\section{RESULTADOS Y DISCUSIÓN}

\section{Registros de temperatura y humedad relativa en laboratorio}

La temperatura fluctuó entre $21,5 \pm 0,8$ y $25,2 \pm 0,8^{\circ} \mathrm{C}$ y un promedio de $24,2 \pm 0,8^{\circ} \mathrm{C}$. La humedad relativa fluctuó entre $29,3 \pm 5,8$ y $51,0 \pm 5,6 \%$ y un promedio de $36,0 \pm 5,9 \%$. Según Mota et al. (2008) las temperaturas que oscilan entre $20^{\circ} \mathrm{C}$ y $30^{\circ} \mathrm{C}$ son adecuadas para el desarrollo de C. arcuatus, lo que sugiere que esta especie está bien adaptada a temperaturas que generalmente se encuentran dentro de invernaderos $o$ en campos abiertos en regiones templadas.

\section{Descripción morfológica de los estados de desarrollo de $C$. arcuatus \\ Huevo}

Los huevos son ovoides, translúcidos, pequeños de 0,43 $\pm 0,04 \mathrm{~mm}$ de longitud y de $0,20 \pm 0,01 \mathrm{~mm}$ de ancho, corium liso y brillante, presenta estrías próximo a la eclosión; carece de procesos microcapilares. Su tamaño es mayor al que posee el huevo de S. phillyreae. Son ovipositados cercanos de las masas cerosas de $S$. phillyreae; solitarios o pueden ser ovipositados dentro de la exuvia de ninfas de S. phillyreae. Próximo a la eclosión aparece en uno de los extremos del huevo unos puntos negros que corresponden a los ocelos de la larva. Existe poca información sobre la descripción del estado de huevo de C. arcuatus (Figuras 1A-B).
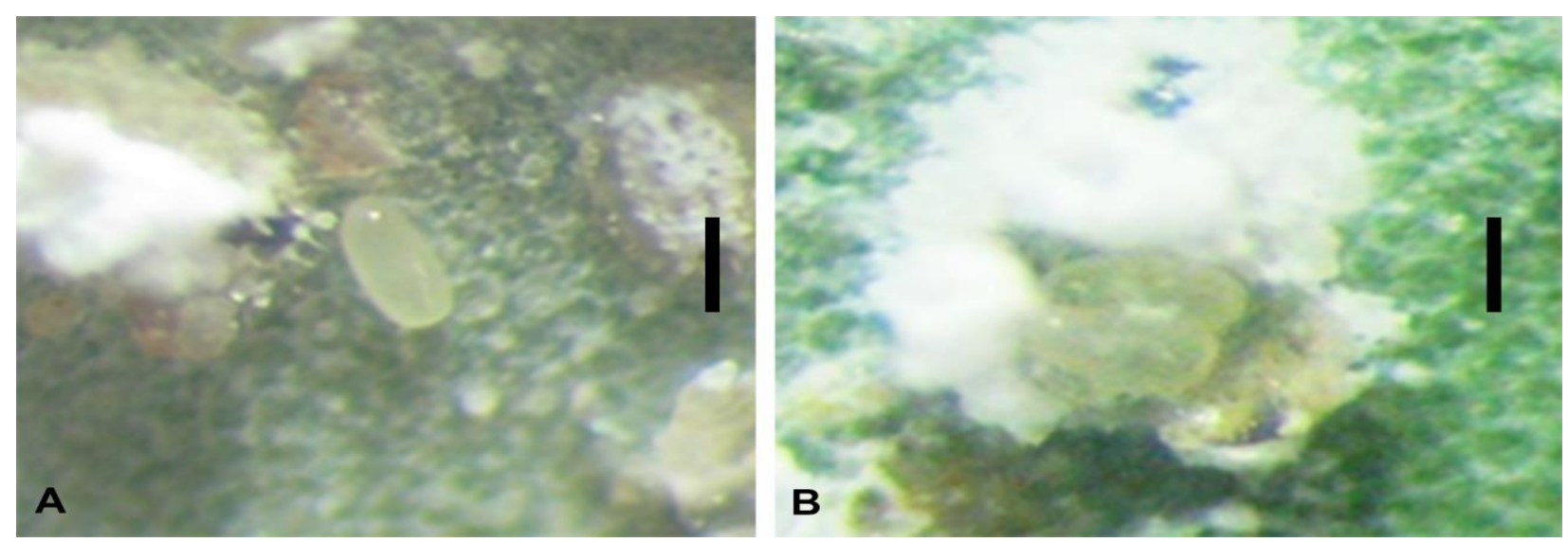

Figura 1. Estado de huevo de Clitostethus arcuatus (Rossi). A. solitario, B. dentro de exuvia de ninfa de S. phillyreae (Haliday). Escala 0,4 mm. 


\section{Larvas}

El estado de larva de $C$. arcuatus presenta cuatro estadíos larvales y corresponde al tipo campodeiforme. El primer estadío larval o larva I es pequeña, de 1,32 \pm $0,12 \mathrm{~mm}$ de longitud y un ancho de cápsula cefálica de $0,12 \pm 0,03 \mathrm{~mm}$. Además, presenta un color blanco translúcido (Figura 2A).

El segundo estadío larval o larva II posee características similares a la larva I; la longitud del cuerpo es de 2,02 $\pm 0,24 \mathrm{~mm}$ y el ancho de la cápsula cefálica es de 0,15 $\pm 0,05 \mathrm{~mm}$. Se observa parte del sistema digestivo pudiéndose observar una mancha rojiza anaranjada en los últimos segmentos que corresponde a parte del sistema digestivo (Figura 2B). El tercer estadío larval o larva III es más robusta que la anterior larva; su color es blanco lechoso con protuberancias negras en el dorso que llevan cerdas que poseen unas gotas de líquido en los ápices. La longitud del cuerpo de 2,7 $\pm 0,15 \mathrm{~mm}$ y la cápsula cefálica posee $0,32 \pm 0,02 \mathrm{~mm}$ de ancho. Ya no se observa la mancha que corresponde al sistema digestivo (Figura 2C).

El cuarto estadío larval o larva IV es el estadío que alcanza la mayor longitud larval, de 2,92 $\pm 0,04 \mathrm{~mm}$ de longitud y con una cápsula cefálica de 0,34 $\pm 0,02 \mathrm{~mm}$ de ancho. Son más notorias las bases del origen de las cerdas dorsales y presentan una coloración negra (Figura 2D). La etapa final de este estadío es la prepupa que es un breve tiempo antes de convertirse en pupa, el individuo reduce su capacidad alimentaria, se inmoviliza y se encorva sobre sí misma reduciendo su longitud (Figura 3A).

La observación de los estadíos larvales concuerda con las afirmaciones de Tavadjoh et al. (2010). De la misma manera, la descripción del estado larval coincide con lo reportado por Gasparini et al. (2007) y Estay et al. (2009). Por otra parte, Soria (2011) sostiene que las larvas recién emergidas son transparentes, luego blancas lechosas, con un punto rojizo en la mitad del abdomen, lo cual según lo observado sería el sistema digestivo visible a través del tegumento de la larva. Sobre el tamaño de las larvas este autor sostiene que son pequeñas, de aproximadamente de 1,5 a 2, 0 mm de longitud; sin embargo, en el presente estudio se obtuvo 2,92 $\pm 0,04 \mathrm{~mm}$ como la longitud máxima para el cuarto estadío larval; la cabeza posee ocelos bien definidos, cuerpo cubierto con abundantes cerdas, abdomen ahusado con 9 segmentos y 3 pares de patas torácicas de color blanco. Se desplazan y camuflan entre las masas cerosas de las ninfas de $S$. phillyreae.

\section{Pupa}

La pupa es de tipo exarate o libre, de 1,8 $\pm 0,13 \mathrm{~mm}$ de longitud, de color blanco y se oscurece próximo a la emergencia del adulto. Además, presenta una mancha oscura en el dorso y cerdas negras con gotas de líquido en las terminaciones que posiblemente le sirven de defensa. Se halla fijada a la hoja por su extremo posterior (Figuras 3A-B). Nuestras observaciones difieren ligeramente de lo registrado por Gasparini et al. (2007) y Estay et al. (2009), así Soria (2011) registró longitudes de 1,5 a 1,8 mm, además indicó que la pupa presenta una mancha rojiza en la mitad del abdomen; esta característica no fue observada. El tamaño de la pupa es el doble comparado con las ninfas de $S$. phillyreae.

\section{Adulto}

De tamaño pequeño, las hembras miden 1,49 $\pm 0,19$ $\mathrm{mm}$ de longitud y $0,93 \pm 0,04 \mathrm{~mm}$ de ancho y los machos de 1,31 $\pm 0,36 \mathrm{~mm}$ de longitud y $0,86 \pm 0,11$ $\mathrm{mm}$ de ancho. La cabeza es prognata, parcialmente oculta por el pronoto y con piezas bucales masticadoras; en hembras, el pronoto es más oscuro en la parte media mientras que en machos es totalmente amarillento con 4 manchas marrones. Ojos compuestos de color negro, antenas clavadas con 11 segmentos y patas de color amarillo pajizo, tarsos trímeros, esto también fue observado por Iqbal et al. (2018). Élitros con variaciones de color, marrón claro a negro, con mancha característica en forma de herradura de color crema claro muy notoria. De acuerdo con González (2006), la coloración de los élitros puede extenderse hasta cubrir la parte central del pronoto; asimismo, Chazeau et al. (1974) y Poussereau et al. (2018) citaron las diversas variaciones de color en esta especie. Machos con cuerpo poco aguzada hacia el extremo posterior y hembras de mayor tamaño y cuerpo globoso; con dimorfismo sexual en la coloración de la cabeza, machos con cabeza amarillenta y hembras con cabeza oscura. Las medidas obtenidas se aproximan a las obtenidas por González (2006), que señala un tamaño de 1,3 a 1,5 mm (Figuras 4A-B). 

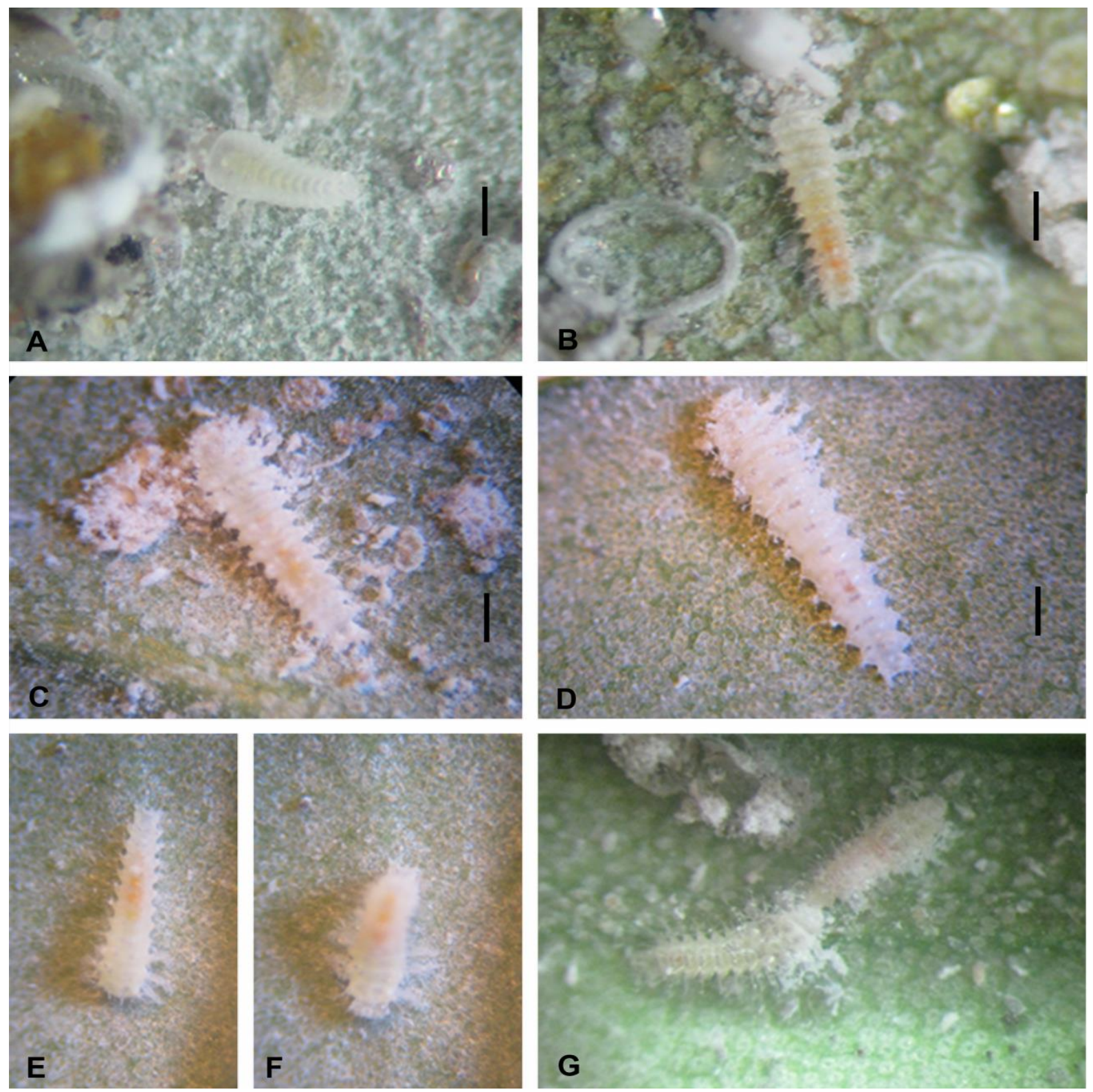

Figura 2. Estado de larva de Clitostethus arcuatus (Rossi). A. Primer estadío larval, B. Segundo estadío larval, C. Tercer estadío larval, D. Cuarto estadío larval; desplazamiento de larva para alimentarse, E. Larva extendida, F. Larva encogida, G. Canibalismo entre larvas. Escala 0,5 mm.

\section{Ciclo de desarrollo de Clitostethus arcuatus \\ Huevo}

El periodo de la incubación de los huevos es de 3-4 días con un promedio de $3,80 \pm 0,75$ que coincide con lo reportado por Tavadjoh et al. (2010), que indica el tiempo de incubación de huevos de $C$. arcuatus de 3,77 $\pm 0,07$ días a $27 \pm 1^{\circ} \mathrm{C}$ en condiciones de laboratorio.

\section{Larva}

El primer estadío larval duró 2,44 \pm 0,50 días (mín. 2 días y máx. 3 días); el segundo estadío larval, 3,52 \pm 0,50 días (mín. 3 días y máx. 4 días); el tercer estadío larval, 4,26 $\pm 0,44$ días (mín. 4 días y máx. 5 días) y el cuarto estadío, 4,52 \pm 0,50 días (mín. 4 días y máx. 5 días). El final del cuarto estadío larval, la prepupa duró 1,04 $\pm 0,20$ días ( 1 día como mín. y 2 como máx.) hasta 
convertirse en pupa. Estos valores comparados con los obtenidos por Tavadjoh et al. (2010): larva I de 2,5 \pm 0,1 días; larva II de 3,5 $\pm 0,1$ días; larva III de 3,4 $\pm 0,1$ días y larva IV de $4,3 \pm 0$, 1días son similares a los obtenidos en este trabajo. La duración total del estado larval fue de 15,78 $\pm 2,13$ días.

\section{Pupa}

El tiempo en estado de pupa duró 4,42 $\pm 0,49$ días (4 días como mín. y 5 días como máx.). Tavadjoh et al. (2010) reportó de 3,9 \pm 0,1 días, valor que difiere con el registrado en este estudio. De acuerdo a estos resultados, el ciclo de desarrollo fue de 24 días lo cual difiere con el reportado por Tavadjoh et al. (2010), que en condiciones de laboratorio a $27 \pm 1^{\circ} \mathrm{C}$ duró $19,1 \pm$ 0,2 días y en condiciones de campo dura 20,2 $\pm 0,1$ días. Sin embargo, Estay et al. (2009) mencionan que el desarrollo de C. arcuatus desde huevo a adulto dura aproximadamente de 15-28 días, influenciado por temperatura y que los estudios realizados en laboratorio muestran que a $28,2^{\circ} \mathrm{C}$ el ciclo de huevo a adulto puede variar de $13-18$ días y a $21,1^{\circ} \mathrm{C}$ alrededor de 28 días, lo que concuerda con lo observado en este trabajo.

\section{Ciclo biológico de Clitostethus arcuatus}

Con los valores obtenidos (ciclo de desarrollo con un promedio de 24 días y la longevidad del adulto que incluye el periodo de pre-oviposición de 3-4 días con un promedio de $3,35 \pm 0,48$ días) se obtuvo el ciclo biológico de C. arcuatus de 77,02 días como promedio. Para el periodo de pre-oviposición, este resultado difiere con lo reportado por Tavadjoh et al. (2010) que mencionan que las hembras apareadas colocaron huevos después de 1,8 $\pm 0,3$ días; sin embargo, el valor obtenido en este estudio para el ciclo biológico se aproxima a lo reportado por este mismo autor que obtuvo 86,91 días para el ciclo biológico (Tablas 1,2).

\section{Parámetros biológicos de Clitostethus arcuatus Capacidad de oviposición}

La capacidad de oviposición promedio fue de 136,85 huevos/hembra y de $3,0 \pm 1,34$ huevos ovipositados/día (2 como mín. y 6 como máx.). Estos resultados difieren con los registrados por Tavadjoh et al. (2010) que reportan un promedio de 181, 83 huevos/hembra y $2,3 \pm 0,3$ huevos ovipositados/día.

\section{Viabilidad de huevos}

De los 112 huevos observados emergieron 80 larvas que representa 71,43\% para la viabilidad de huevos; $y$ una diferencia del 28,57\% para los huevos no eclosionados.

\section{Mortalidad de larvas}

De las 80 larvas del primer estadío larval, sólo 51 pasaron al siguiente estadío, que representa el 63,75\% y la diferencia de $36,25 \%$ para la mortalidad determinada. De las 51 larvas del segundo estadío larval, todas las larvas pasaron al tercer estadío. En los estadíos II, III y IV no se observó muertes por lo tanto lograron completar todo su desarrollo hasta el estado de pupa y posteriormente hasta adultos.
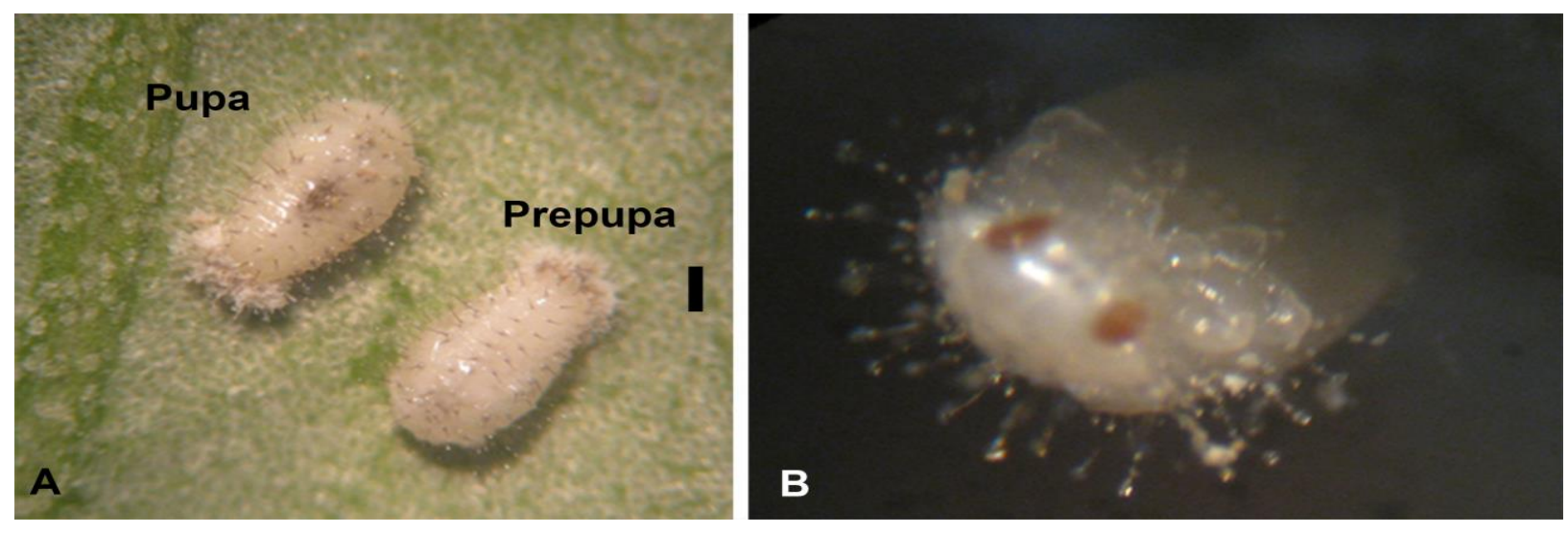

Figura 3. Estado de pupa de Clitostethus arcuatus (Rossi). A. Pupa y prepupa, B. Vista ventral. Escala 0,5 mm. 

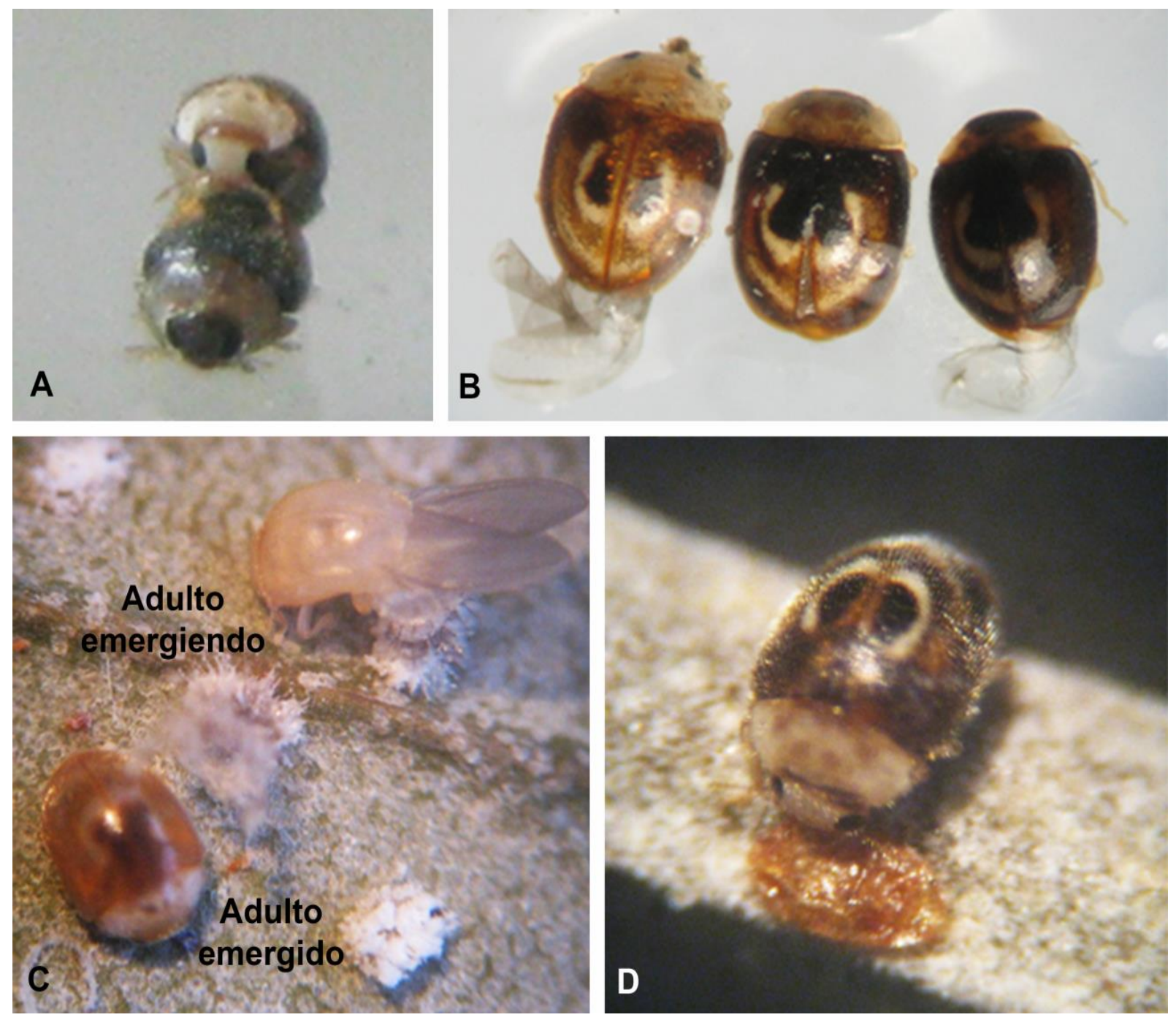

Figura 4. Adultos de Clitostethus arcuatus (Rossi). A. Dimorfismo sexual, cópula, B. Variación de color, C. Emergencia de adulto, D. Adulto alimentándose de queresa.

Tabla 1. Ciclo de desarrollo y longevidad de Clitostethus arcuatus (Rossi) en los meses de abril a julio, 2013.

\begin{tabular}{lccc}
\hline Estado de desarrollo & Mínimo (días) & Máximo (días) & Promedio \\
\hline Huevo & 3 & 4 & $3,80 \pm 0,75$ \\
Larva I & 2 & 3 & $2,44 \pm 0,50$ \\
Larva II & 3 & 4 & $3,52 \pm 0,50$ \\
Larva III & 4 & 5 & $4,26 \pm 0,44$ \\
Larva IV & 4 & 5 & $4,52 \pm 0,50$ \\
Prepupa & 1 & 2 & $1,04 \pm 0,20$ \\
Pupa & 4 & 5 & $4,42 \pm 0,49$ \\
Huevo a Adulto & 21 & 28 & $24,0 \pm 0,48$ \\
Longevidad de Hembra & 45 & 64 & $55,50 \pm 5,71$ \\
Longevidad de Macho & 40 & 60 & $50,90 \pm 6,51$ \\
\hline
\end{tabular}


Tabla 2. Ciclo de desarrollo y Ciclo biológico de Clitostethus arcuatus (Rossi) en los meses de abril a julio, 2013.

\begin{tabular}{|c|c|c|c|c|c|}
\hline Estados & Estadíos & $\begin{array}{c}\text { Duración de } \\
\text { estadíos (días) }\end{array}$ & $\begin{array}{c}\text { Duración de } \\
\text { estados (días) }\end{array}$ & $\begin{array}{c}\begin{array}{c}\text { Ciclo de desarrollo } \\
\text { (días) }\end{array} \\
\end{array}$ & Ciclo biológico (días) \\
\hline Huevo & & & 3,80 & \multirow{7}{*}{24} & \\
\hline \multirow{5}{*}{ Larva } & I & 2,44 & & & \\
\hline & II & 3,52 & & & \\
\hline & III & 4,26 & 15,78 & & \\
\hline & IV & 4,52 & & & \\
\hline & Prepupa & 1,04 & & & \\
\hline Pupa & & & 4,42 & & \\
\hline \multirow{2}{*}{ Adulto } & \multicolumn{2}{|c|}{ Pre-oviposición } & 3,35 & & \\
\hline & \multicolumn{2}{|c|}{ Longevidad } & 49,67 & & 77,02 \\
\hline
\end{tabular}

Estos resultados nos indican que sólo en el primer estadío larval se presenta mortalidad, ya que es el más sensible y con desplazamiento reducido, esto disminuyó en los estadíos siguientes; lo cual coincide con Tavadjoh et al. (2010) que reportan que el primer estadío larval tuvo la mayor tasa de mortalidad, pues es la etapa más vulnerable de $C$. arcuatus; además, sostiene que la mortalidad de esta especie se estabiliza después del segundo estadio larval.

\section{Capacidad de predación}

En la Tabla 3 se observan los resultados obtenidos al ser ofrecidos huevos y ninfas de $S$. phillyreae a las larvas de $C$. arcuatus, siendo observada la preferencia de huevos por las larvas I y II, esto debido posiblemente a su tamaño pequeño y sus movimientos lentos; mientras que la larva III y IV depredaron huevos y ninfas; adultos de C. arcuatus depredaron huevos, ninfas y adultos. Los resultados obtenidos para el primer estadio difieren con lo registrado por Tavadjoh et al. (2010) ya que señaló que el primer estadío larval de $C$. arcuatus se alimenta de huevos y ninfas; sin embargo, en este estudio, las larvas I y II sólo depredaron huevos. Asimismo, estos mismos autores sostienen que el segundo, tercer y cuarto estadio larval de $C$. arcuatus se alimentan de todos los estadios de $S$. phillyreae, pero con preferencia de huevos; fue observado que las larvas III y IV depredan huevos y ninfas, y que la larva IV depredó la mayor cantidad de huevos. En otro reporte, Sepúlveda et al. (2011) sostienen que la capacidad de depredación de adultos de $C$. arcuatus a ninfas de $S$. phillyreae fue 6,6 de ninfas/día mientras que en el presente trabajo se obtuvo 4,6 ninfas/día. Otro autor, Estay et al. (2009) mencionan que tanto la larva y adultos de C. arcuatus son capaces de comer huevos, ninfas y también adultos de S. phillyreae, sin embargo, al estar presentes todos los estados, prefiere huevos y ninfas; lo cual se corrobora en este estudio.

\section{Proporción sexual}

La proporción sexual de $C$. arcuatus fue de 1:1,59 (macho: hembra) lo que indica un valor ligeramente superior en hembras, lo cual difiere con lo reportado por Tavadjoh et al. (2010) que señalan que en condiciones de laboratorio a $27 \pm 1^{\circ} \mathrm{C}$, la proporción sexual es 1:1,38 (hembra: macho) siendo el número de machos ligeramente mayor que el número de hembras.

Tabla 3. Capacidad de depredación de los estados de desarrollo de Clitostethus arcuatus (Rossi) a huevos, ninfas y adultos de Siphoninus phillyreae (Haliday) por día, 2013.

\begin{tabular}{|c|c|c|c|c|}
\hline \multicolumn{2}{|c|}{ Estado } & $\begin{array}{c}\text { Promedio de huevos } \\
\text { depredados/día }\end{array}$ & $\begin{array}{c}\text { Promedio de ninfas } \\
\text { depredadas/día }\end{array}$ & $\begin{array}{c}\text { Promedio de adultos } \\
\text { depredados/día }\end{array}$ \\
\hline \multirow{4}{*}{ Larva } & I & 3,8 & 0 & 0 \\
\hline & II & 5 & 0 & 0 \\
\hline & III & 8,2 & 3,6 & 0 \\
\hline & IV & 11,4 & 3,4 & 0 \\
\hline \multicolumn{2}{|c|}{ Adulto } & 9,8 & 4,6 & 1,4 \\
\hline
\end{tabular}




\section{Longevidad}

La longevidad de los adultos de C. arcuatus fue de 53,02 \pm 6,69 días (mín. 40 días y máx. 64 días), las hembras vivieron $55,50 \pm 5,71$ y los machos $50,90 \pm$ 6,51 días, esto difiere con lo obtenido por Tavadjoh et al. (2010) que indican que a $27 \pm 1{ }^{\circ} \mathrm{C}$ es $62-73$ días para los adultos de $C$. arcuatus y la longevidad de las hembras (73 días) es mayor que la de los machos (62 días). Asimismo, Estay et al. (2009) señalan que el adulto de $C$. arcuatus tiene una larga vida, llegando muchos de ellos hasta el año siguiente para reiniciar el proceso de reproducción.

\section{Comportamiento de Clitostethus arcuatus Emergencia del adulto}

La emergencia del adulto es lenta, emerge por la parte dorsal del tegumento pupal, del cual logran salir ayudándose de las mandíbulas y patas, luego permanecen inmóviles por algunos minutos. Al emerger, el adulto no tiene una coloración marcada, posee un color marrón claro y las manchas elitrales son poco notorias (Figura 4C).

\section{Desplazamiento de larvas}

El cuerpo de las larvas giba para desplazarse (Figuras 2E-F). La larva I posee un movimiento restringido a la hoja y come lo que logra alcanzar, el movimiento de la larva II es similar al anterior; larva III y IV realizan mayor movimiento que larva I y II, éstas logran trasladarse de una hoja a otra. Cuando el cuarto estadío larval se prepara para empupar reduce sus movimientos.

\section{Alimentación}

Tanto larvas y adulto de $C$. arcuatus capturan a su presa ayudándose de sus patas anteriores y después la devoran con sus piezas bucales masticadoras; succionan el contenido de los huevos y devoran ninfas y adultos de $S$. phillyreae. Las larvas I y II prefieren huevos de $S$. phillyreae y en menor cantidad, ninfas. Los adultos de $C$. arcuatus también fueron observados alimentándose de queresas (Figura 4D). Se observó canibalismo entre larvas de $C$. arcuatus, también reportado por Tavadjoh et al. (2010).

\section{Apareamiento}

La cópula se realizó al tercer o cuarto día después de la emergencia. Para el apareamiento, el macho de $C$. arcuatus se aproxima a la hembra, cogiendo con sus patas anteriores a la hembra a la altura del abdomen, se apoyan en la parte anterior de los élitros y las patas medias sobre la superficie de los élitros, a la vez que la parte caudal del abdomen se dobla hacia la parte ventral caudal del abdomen de la hembra. La cópula dura entre 60 a 70 minutos (Figura 4A). Tavadjoh et al. (2010) reportan que la cópula duró aproximadamente 67.1 minutos, lo cual se encuentra dentro del rango registrado en este estudio.

\section{Oviposición}

Los huevos fueron ovipositados dentro y fuera de las exuvias de ninfas de $S$. phillyreae (Figuras 1A-B), esto concuerda con lo reportado por Tavadjoh et al. (2010) que mencionan que los huevos son ovipositados entre las masas cerosas de $S$. phillyreae. Sin embargo, en este estudio también se observó huevos solitarios en hojas sin presencia cercana de presas.

\section{CONCLUSIONES}

Para contribuir al conocimiento de la biología de Clitostethus arcuatus (Rossi, 1794) se determinó su ciclo biológico que fue de 77,02 días y sus parámetros biológicos: capacidad de oviposición de 136,35 huevos/hembra; viabilidad de huevos $71,43 \%$; mortalidad de larvas 36,25\% sólo para LI; capacidad de depredación de LI 3,8 huevos/día y LII 5 huevos/día de S. phillyreae; LIII 8,2 huevos/día y 3,6 ninfas/día de S. phillyreae; LIV 11,4 huevos/día y 3,4 ninfas/día de S. phillyreae; y adultos 9,8 huevos/día, 4,6 ninfas/día y 1,4 adultos/día de $S$. phillyreae; proporción sexual de 1:1,59 (macho: hembra) y longevidad de adulto 53,02 días.

Se recomienda realizar estudios futuros evaluando presencia, poblaciones, registros de presas y preferencias alimenticias de este depredador en campo, así como la posibilidad de realizar evaluaciones de depredación con otras especies de mosca blanca que afectan otros cultivos de importancia económica.

\section{Conflictos de intereses}

Los autores firmantes del presente trabajo de investigación declaran no tener ningún potencial conflicto de interés personal o económico con otras personas $\mathrm{u}$ organizaciones que puedan influir indebidamente con el presente manuscrito. 


\section{Contribuciones de los autores}

Preparación y ejecución: JMCS, JGMM; Desarrollo de la metodología: JMCS, JGMM; Concepción y diseño: JMCS, JGMM; Edición del artículo: JMCS; Supervisión del estudio: JGMM.

\section{REFERENCIAS}

- Almonte, E., Alcócer, S., \& Ccaso, M. (2012). Mosca Blanca en Olivo. Proyecto: "Mejoramiento del control integrado de Mosca Blanca Siphoninus phillyreae - Mosca Blanca en el cultivo del olivo en las Provincias de Islay y Caravelí". Boletín Técnico Informativo, 1 (1).

- Bellows, T., Meisenbancher, C., Paine, T., Leddy, P., Arakawa, K. \& Kabasshima, J. (1990). Biological control sought for ash whitefly. California Agriculture, 44 (1), 4-6.

- Chazeau J., Étienne, J. \& Fürsch, H. (1974). Les Coccinellidae de l'île de la Réunion. Bulletin du Muséum National d' Histoire Naturelle 3e série (210) Zoologie, 140, 265-297.

- Churata Salcedo, J. M. (2013). Ciclo y parámetros biológicos de Clitostethus arcuatus Rossi (Coleoptera: Coccinellidae), depredador de mosca blanca, en condiciones de laboratorio. Tesis de pregrado para optar el Título Profesional de Biólogo, Universidad Nacional de San Agustín, Arequipa, Perú.

- Estay, P., González, V. \& Rojas, C. (2009). Mosquita Blanca del Fresno, Siphoninus phillyreae (Hemiptera: Aleyrodidae) y su Controlador Biológico: la Chinita Predadora, Clitostethus arcuatus (Coleoptera: Coccinellidae), en olivo. Informativo $\mathrm{N}^{\circ}$ 8. Chile. INIA - Ururi.

- Gasparini, M., Holgado, M. \& Rodríguez, F. (2007). Presencia de Clitostethus arcuatus (Coleoptera: Coccinellidae) sobre olivos infestados con Siphoninus phillyreae (Hemiptera: Aleyrodidae) en Argentina. Revista de la Sociedad Entomológica Argentina, 66 (1-2), 169-170.

- González, G. (2012). Coccinellidae de Perú. Disponible en: $<$ http://www.coccinellidae.cl/paginasWebPeru/Pa ginas/Clitostethus_arcuatus_Peru.php>

- Haliday, A. H. (1835). Aleyrodes phillyreae. Entomology Magazine, 2: 119-120.
- Iqbal, Z., Farooq, M., Bodlah, I. \& Szawaryn, K. (2018). Review of Clitostethus Weise, Parastethorus Pang et Mao and Stethorus Weise (Coleoptera: Coccinellidae) from Pakistan. Oriental Insects, 53:3,340-355. DOI: 10.1080/00305316.2018.1492987

- Mota, J. A., Onofre, A. \& Ventura, P. (2008). Temperature dependence for development of the whitefly predator Clitostethus arcuatus (Rossi). BioControl, 53, 603-613. DOI 10.1007/s10526007-9101-5

- Osis, V. (1999). Determinación y ciclo biológico de Siphoninus phillyreae (Haliday) Homoptera: Aleyrodidae "Mosca blanca de Fresno" en la zona urbana de Arequipa. Tesis para optar el Título Profesional de Biólogo, Universidad Nacional de San Agustín, Arequipa, Perú.

Pedemonte, M., Bruno, M., \& Grilli, M. (2009). Influencia de las características del entorno y la abundancia de Clitostethus arcuatus sobre poblaciones de Mosquita Blanca en cultivos de olivo. XVII Jornadas Científicas de la Sociedad de Biología de Córdoba.

Poussereau, J., Coutanceau J.P., Nicolas, V. \& Gomy, Y. (2018). Les coccinelles de l'île de la Réunion. Orphie, Reunion.

Rodríguez, F. \& Rodríguez, S. (2005). Control biológico de Plagas Urbanas. INIA - La Cruz. Chile.

Rossi, P. (1794). Mantissa Insectorum, Tome II. Pisis.

SENASA [Servicio Nacional de Sanidad Agraria]. (2010). Informe: Prospección de mosca blanca en el cultivo del olivo.

Sepúlveda, G., Bobadilla, D., Gallo, P., Vargas, H., Bilbao, C. \& Maldonado, M. (2011). Metodología utilizada para la crianza y reproducción de Clitostethus arcuatus (Rossi). Departamento de Recursos Ambientales. Facultad de Ciencias Agronómicas. Universidad de Tarapacá. Chile.

Soria, J. (2011). Evaluación e identificación de la fauna benéfica nativa controladora de la mosca blanca del fresno Siphoninus phillyreae Haliday, 1835 (Hemiptera: Aleyrodidae) en olivos de la Región Tacna. Tesis de pregrado para optar el título Profesional de Biólogo - Microbiólogo. Universidad Nacional Jorge Basadre Grohmann, Tacna - Perú. 85 p. 
- Tapia, F., Ibacache, A., Sierra, C., Larraín, P., Riveros, F., \& Martínez, L. (2009). Seminario Manejo Agronómico Industrial Olivícola. Instituto de Investigaciones Agropecuarias Centro Regional de Investigaciones Intihuasi. Chile.

- $\quad$ Tavadjoh, Z., Hamzehzarghani, H., Alemansoor, H., Khalghani, J., \& Vikram, A. (2010). Biology and feeding behaviour of ladybird, Clitostethus arcuatus, the predator of the ash whitefly, Siphoninus phillyreae, in Fars province, Iran.
Journal of Insect Science, 10: 120. Disponible en: insectsicence.org/10.120

- Valencia, L. (2011). Reestablecimiento de Siphoninus finitimus Silvestri (Hemiptera: Aleyrodidae) como una especie válida, en base a datos morfológicos, plantas hospederas y parasitoides en el Perú. Revista Peruana de Entomología, 46 (2), 59-67. 